\title{
AMSU-A Land Surface Emissivity Estimation for Numerical Weather Prediction Assimilation Schemes
}

\author{
CATHERINe PRigent \\ Laboratoire d'Etudes du Rayonnement et de la Matière en Astrophysique, Centre National de la Recherche Scientifique, \\ Observatoire de Paris, Paris, France \\ FrédéRIC Chevallier* \\ European Centre for Medium-Range Weather Forecasts, Reading, United Kingdom \\ FATIMA KARBOU \\ Centre National de la Recherche Scientifique, Centre d'Etude des Environnements Terrestre et Planétaires, Vélizy, France \\ Peter Bauer and Graeme Kelly \\ European Centre for Medium-Range Weather Forecasts, Reading, United Kingdom
}

(Manuscript received 14 June 2004, in final form 23 September 2004)

\begin{abstract}
This study describes the work performed at the European Centre for Medium-Range Weather Forecasts (ECMWF) to estimate the microwave land surface emissivities at Advanced Microwave Sounding Unit (AMSU)-A frequencies within the specific context and constraint of operational assimilation. The emissivities are directly calculated from the satellite observations in clear-sky conditions using the surface skin temperature derived from ECMWF and the Radiative Transfer for the Television and Infrared Observation Satellite Operational Vertical Sounder (RTTOVS) model, along with the forecast model variables to estimate the atmospheric contributions. The results are analyzed, with special emphasis on the evaluation of the frequency and angular dependencies of the emissivities with respect to the surface characteristics. Possible extrapolation of the Special Sensor Microwave Imager (SSM/I) emissivities to those of the AMSU is considered. Direct calculation results are also compared with emissivity model outputs.
\end{abstract}

\section{Introduction}

Since 1998, the Advanced Microwave Sounding Unit (AMSU)-A on board the National Oceanic and Atmospheric Administration (NOAA) polar orbiters provide unique atmospheric temperature profiling capabilities, both in the troposphere and in the stratosphere. Exploiting these data has been a key challenge for numerical weather prediction (NWP) centers (e.g., English et al. 2000; Kelly and Bauer 2000).

\footnotetext{
* Current affiliation: Centre National de la Recherche Scientifique, Laboratoire des Sciences du Climat et l'Environnement, Gif-sur-Yvette, France.
}

Corresponding author address: Dr. Catherine Prigent, Centre National de la Recherche Scientifique, Laboratoire d'Etudes du Rayonnement et de la Matiere en Astrophysique, Observatoire de Paris, 61, Avenue de l'Observatoire, Paris, France.

E-mail: catherine.prigent@obspm.fr
Several factors contribute to make assimilation of such data more difficult over land than over ocean. Microwave land surface emissivities are usually much higher than ocean emissivities, making the surface contribution larger. They have also a shorter spatial correlation scale. In addition, they are very complex to model, from arid surfaces to dense vegetation or snow, because they are dependent on a large number of highly variable parameters. The impact of the emissivity errors on the atmospheric temperature and humidity retrievals has been analyzed by English (1999). Efforts have been directed toward a better understanding of the mechanisms that are responsible for the microwave emission of continental surfaces, from both theoretical analysis and field experiments. Model developments include detailed simulations of bare soil (e.g., Shi et al. 2002), vegetation canopy (Karam et al. 1992; Ferrazoli et al. 2000), and snow (Fung 1994). Truck-mounted radiometers (Matzler 1990) and airborne instruments (Calvet et al. 1996; Wigneron et al. 1997; Hewison and 
English 1999; Hewison 2001) provide some in situ estimates of the emissivities to help to anchor the models. However, even assuming that a perfect land surface emissivity model exists, would the inputs it will require (soil texture and humidity, vegetation characteristics, percentage of vegetation coverage within a field of view, and snow density, to name only a few) be available on a global basis with a resolution that is compatible with the satellite resolution and with the required accuracy?

Very few groups have so far examined the problem of global microwave land surface emissivities as a first step for data assimilation over the continents.

Global land surface emissivity maps were first produced at Special Sensor Microwave Imager (SSM/I) frequencies by Prigent et al. $(1997,1998)$ by removing the contribution of the atmosphere, clouds, and rain using ancillary data. The emissivities are estimated for SSM/I observation conditions, that is, for a $53^{\circ}$ zenith angle at $19.35,22.235,37.0$, and $85.5 \mathrm{GHz}$. These emissivities have been the basis for a large number of studies-first, to analyze the land surface characteristics like vegetation (Prigent et al. 2001a) or inundation (Prigent et al. 2001b; Fily et al. 2003), or to help to retrieve the surface skin temperature and atmospheric parameter over land (Prigent et al. 1999; Aires et al. 2001; Prigent et al. 2003). Frequency and angular inter-/ extrapolations of these SSM/I emissivities for the Special Sensor Microwave Temperature Profiler (SSM/ T)-1 and AMSU-A application have been suggested (Prigent et al. 2000) but have not been used so far in an operational context. Similar direct calculations of the emissivities have also been performed by Felde and Pickle (1995) and Jones and Vonder Haar (1997) for limited geographical regions.

In a different way, Weng et al. (2001) chose to develop a global model to estimate the emissivity for the various surface conditions encountered over the continents, using different radiative transfer solutions depending on the surface characteristics. Model inputs are provided by a land surface model, such as the one in the Global Data Assimilation System of the National Centers for Environmental Prediction (NCEP). The simulations have been compared with both ground-based measurements and with emissivities directly calculated from AMSU-A observations.

As described by Kelly and Bauer (2000), the current forecast system at the European Centre for MediumRange Weather Forecasts (ECMWF) uses an intermediate approach to assimilate 10 of the AMSU-A channels, 2 of which have a weak contribution from the surface (at 53.596 and $54.4 \mathrm{GHz}$ ). In this approach, the land surface emissivity is obtained from the observations by a parametric model after identification of the scene (Grody 1988). Despite the positive impact of the two AMSU-A surface-affected channels on the forecast quality (Kelly and Bauer 2000), the treatment of the surface emissivity is too simple to allow the extension of the approach to channels that are more affected by the surface, like at $52.8 \mathrm{GHz}$.

This study describes the work performed at ECMWF to apply the Prigent et al. (1997, 1998) approach to estimate the microwave land surface emissivities within the specific context and constraint of operational assimilation.

This paper is organized as follows. The method used at ECMWF to calculate directly the land surface emissivities for AMSU-A observation is described (section 2). The results are then analyzed, with special emphasis on the evaluation of the frequency and angular dependencies of the emissivities with respect to the surface characteristics. Possible extrapolation of the SSM/I emissivities to those of the AMSU is considered. Direct calculation results are also compared with emissivity model outputs. The last section offers conclusions on the method that is selected.

\section{Direct calculation of the land surface emissivities for the AMSU-A frequencies and observation conditions}

The method adopted to calculate directly the land surface emissivities at AMSU-A frequencies and observation conditions follows closely the scheme previously developed for SSM/I (Prigent et al. 1997, 1998). It uses 1) the AMSU-A observed brightness temperatures, 2) the Radiative Transfer for the Television and Infrared Observation Satellite (TIROS) Operational Vertical Sounder (TOVS) (RTTOV) (Eyre 1991; Saunders et al. 1999) atmospheric radiation model, and 3) the ECMWF short-range forecasts.

\section{a. The method}

\section{1) BASIC PRINCIPLE}

Over a flat lossy surface, the integrated radiative transfer equation in the Rayleigh-Jeans approximation for a nonscattering plane-parallel atmosphere can be expressed in terms of brightness temperature for a given polarization state $p$ :

$$
\mathrm{Tb}_{p}=T_{\text {surf }} \epsilon_{p} e^{-\tau(0, H) / \mu}+T_{\mathrm{atm}}^{\downarrow}\left(1-\epsilon_{p}\right) e^{-\tau(0, H) / \mu}+T_{\mathrm{atm}}^{\uparrow},
$$

with $T_{\mathrm{atm}}^{\downarrow}=\int_{H}^{0} T(z) \alpha(z) \exp [-\tau(z, 0) / \mu] d z$ and $T_{\mathrm{atm}}^{\uparrow}=$ $\int_{0}^{H} T(z) \alpha(z) \exp [-\tau(z, H) / \mu] d z$. Here $\mathrm{Tb}_{p}$ is the brightness temperature measured by the satellite for polarization state $p ; T_{\text {surf }}$ is the surface "skin" temperature; $\epsilon_{p}$ is the surface emissivity for polarization state $p ; \mu=$ $\cos (\theta)$, where $\theta$ is the incidence angle on the surface; $\alpha(z)$ is the atmospheric absorption by gases at altitude $z ; T(z)$ is the atmospheric temperature at altitude $z$; $\tau\left(z_{0}, z_{1}\right)=\int_{z_{0}}^{z_{1}} \alpha(z) d z$ is the atmospheric extinction from $z_{0}$ to $z_{1}$; and $H$ is the orbiter height.

This equation leads to 


$$
\epsilon_{p}=\frac{\mathrm{Tb}_{p}-T_{\mathrm{atm}}^{\uparrow}-T_{\mathrm{atm}}^{\downarrow} e^{-\tau(0, H) / \mu}}{e^{-\tau(0, H) / \mu}\left(T_{\text {surf }}-T_{\mathrm{atm}}^{\downarrow}\right)} .
$$

At AMSU-A frequencies, the radiation emanates from only a thin surface layer of bare soil and water with the penetration depth of the order of the wavelength in soil and less for water. That leads to the following assumptions: there is no volume scattering, the surface temperature is the skin temperature, and for flat surfaces the reflection is considered to be specular. However, volume scattering is involved in the cases of vegetation, snow cover, or very dry sand because the microwave radiation can arise from below and within the canopy or snow layer. When the terrain is rough on scales between the radiation wavelength and the size of the field of view, the surface acts as a set of scattering facets with a complex distribution of orientations. In these cases Eqs. (1) and (2) involve some "effective" emissivity and temperature, aggregated over the depth of penetration and the field of view of the satellite instrument (English and Hewison 1998; Deblonde 2000).

For simplicity in the following discussion, the term surface emissivity will be used instead of "effective" surface emissivity.

The method consists in solving the radiative transfer equation in Eq. (2) for the surface emissivity for each channel using ancillary data to specify the atmospheric and other surface parameters.

\section{2) VALIDITY OF THE SPECULAR APPROXIMATION}

The validity of the specular approximation has been evaluated by comparing the difference between obtained emissivities using the specular approach and emissivities calculated assuming a rougher surface (Fig. $1)$. For the rougher case, the downwelling radiations come from a cone centered on the specular angle with a $10^{\circ}$ Gaussian distribution. The simulations have been conducted for specular emissivities from 0.7 to 1 , and for observation angles up to $60^{\circ}$. Results are shown for a U.S. standard atmosphere (integrated water vapor content of $14 \mathrm{~kg} \mathrm{~m}^{-2}$ ). For this atmosphere at nadir the atmospheric transmission is $0.92,0.95,0.69$, and 0.83 at $23,31,50$, and $89 \mathrm{GHz}$ respectively. The obtained emissivity differences are small. Even for high observation angles the errors related to the specular approximation are within $1 \%$ for all channels.

\section{3) Application to AMSU-A data at ECMWF}

The AMSU-A emissivity calculations are performed using the ancillary data provided by the ECMWF shortrange $(0-12 \mathrm{~h})$ forecasts at the same location and time as the satellite observations. In a first step, cloudy observations are eliminated. In comparison with what has been done for SSM/I previously (Prigent et al. 1997, 1998), the main difference arises from the fact that the cloud, surface skin, and atmospheric products are not derived from the International Satellite Cloud Climatology Project (ISCCP; Rossow and Schiffer 1999). The ISCCP data are not available in real time, which prevents their use in NWP operational assimilation schemes. Here the selection of the clear pixels is based on the forecast model, that is, the observations that correspond to a nonzero fractional area cloud cover in the model are excluded. The atmospheric contribution is then calculated from the forecast model variables and the RTTOV model. Last, with the forecast model surface temperature, the "effective" surface emissivity is calculated from Eq. (2).

Whatever the ancillary information source is, its errors affect the emissivity retrieval. Prigent et al. (1997) discuss the emissivity errors when using the ISCCP data and conclude in favor of a $1 \%$ accuracy for the retrieved emissivities. When using ECMWF data, the error caused by the atmospheric products is limited, because the ECMWF analysis interpolated to $\pm 30 \mathrm{~min}$ from the satellite observations is used. This is not the case for the error coming from the cloud and surface skin temperature products, because no such observations are yet assimilated at ECMWF; these variables are indirectly constrained by other observation types (like wind sensors for clouds). Still, the skin temperature error lies within the 4-K figure used in the Prigent et al. (1997) study, except in very dry regions (Trigo and Viterbo 2003). Also, cloud occurrence in the ECMWF short-range forecast is realistic if we except convection regions (Chevallier and Kelly 2002). In summary, the $1 \%$ accuracy previously quoted is expected to apply to the current computations, except in some regions such as deserts and the intertropical convergence zone, where biases are likely to occur.

\section{b. Brief description of the AMSU-A instrument}

The AMSU-A instrument is described in Diak et al. (1992) and Saunders (1993). It is a cross-track-scanning instrument with 30 scan positions at $3.3^{\circ}$ intervals from $-14.5 \times 3.3^{\circ}$ to $+14.5 \times 3.3^{\circ}$, which translate into local zenith angles $\theta_{z}$ up to $58.5^{\circ}$. The spatial resolution is 50 $\mathrm{km}$ at nadir. Table 1 summarizes the AMSU-A characteristics and gives the total atmospheric transmission at nadir for each channel for two standard atmospheres. The polarization measured by AMSU-A rotates with scan angle because of the rotating-reflector/fixed-feed type of antenna design. If $\theta_{s}$ is the scan angle and $\theta_{z}$ is the local zenith angle, then the AMSU-A surface emissivity $\epsilon\left(\theta_{z}\right)$ seen for a local zenith angle $\theta_{z}$ is given by

$$
\epsilon\left(\theta_{z}\right)=\epsilon_{p}\left(\theta_{z}\right) \cos ^{2}\left(\theta_{s}\right)+\epsilon_{q}\left(\theta_{z}\right) \sin ^{2}\left(\theta_{s}\right)
$$

$\epsilon_{p}\left(\theta_{z}\right)$ and $\epsilon_{q}\left(\theta_{z}\right)$ are the two orthogonal polarized surface emissivities at $\theta_{z}$ local zenith angle. Depending on the channels, $p$ will represent the vertical or the horizontal polarization. The polarization $p$ seen when the 

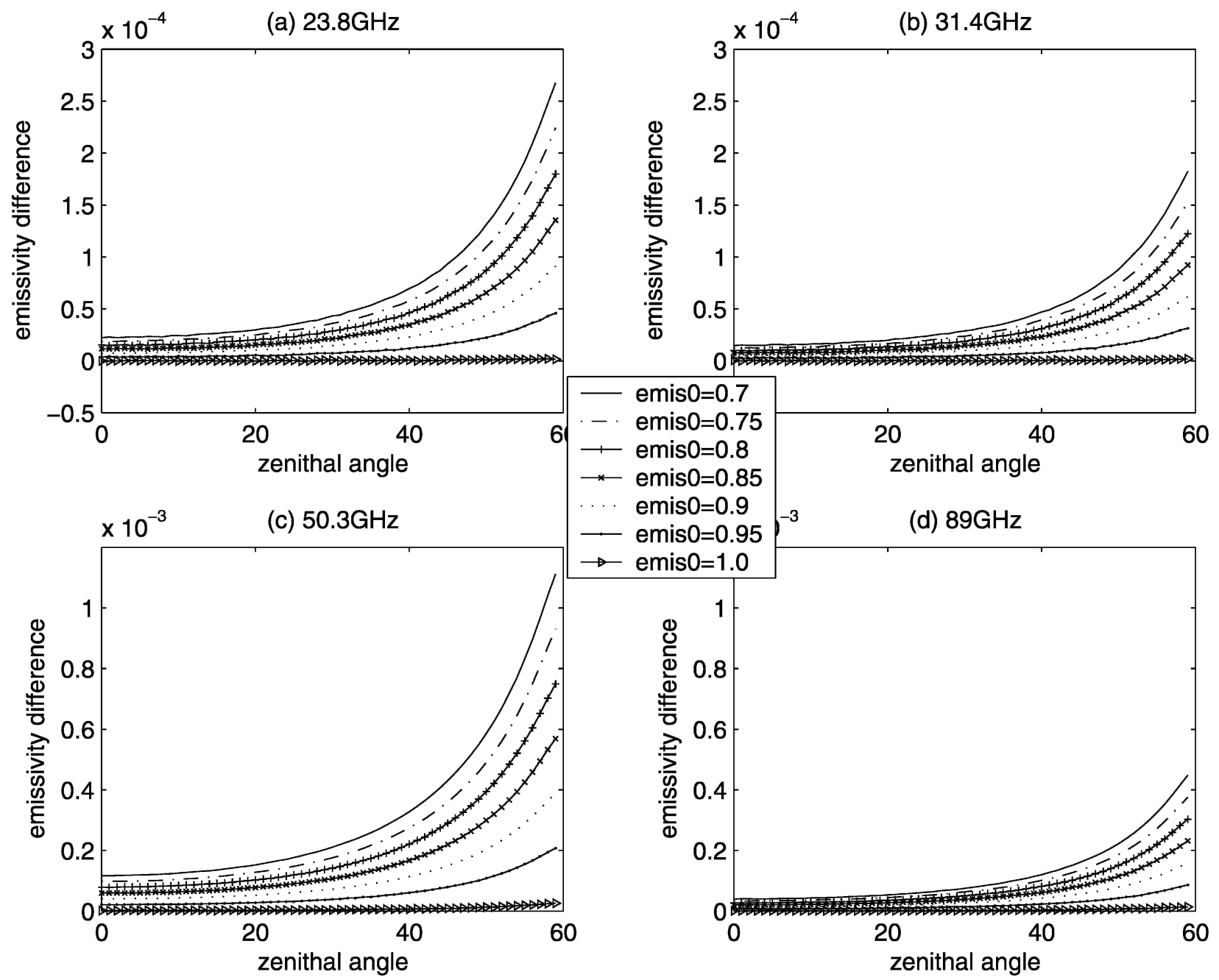

FIG. 1. The difference between specular and pseudo-Lambertian calculations for four AMSU-A frequencies and for surface emissivities varying between 0.7 and 1 . For the rougher surface, the downwelling radiation comes from a cone centered on the specular angle with a $10^{\circ}$ Gaussian distribution, whereas in the specular approximation the calculation only considers the downwelling radiation in the specular direction.

incidence is close to nadir (i.e., for $\theta_{z}=\theta_{s}$ very close to $\left.0^{\circ}\right)$ is indicated for each channel on Table 1.

\section{c. Emissivity maps}

Calculations are performed globally for 2 monthsJuly 2002 and January 2003-with data from NOAA$15,-16$, and -17 . They have been mapped to the forecast model regular 40-km grid (T511 spectral truncature).

Figure 2 shows the average number of AMSU-A observations per grid cell for two incidence angle ranges $\left(10^{\circ}-20^{\circ}\right.$ and $\left.40^{\circ}-50^{\circ}\right)$, for clear and cloudy scenes together (x), and for cloud-free observations only (o). Results are presented for July 2002. For other months, the amount of cloud cover changes with latitude, but still the number of observations per grid cell and per incidence angle can be small (less than four). We checked that the results presented in the following are not significantly affected by relaxing the cloud detection, that is, letting observations with up to $5 \%$ model low and medium cloud cover and allowing any amount of model high clouds.

Figure 3 shows the mean emissivities for four frequencies for two incidence angle ranges $\left(10^{\circ}-20^{\circ}\right.$ on the left and $40^{\circ}-50^{\circ}$ on the right). For the channels with atmospheric transmission lower than $\sim 20 \%$ at nadir, the emissivity estimates are very noisy (not shown); the surface contribution to the observed signal is limited and errors in the radiative transfer model or in the atmospheric profiles have a larger impact on the emissivity calculation. Because the expected spectral variation of the emissivities is limited, extrapolations of the emissivities to the other channels in the $\mathrm{O}_{2}$ band will be valid. The calculated emissivities show consistent spatial structures that can be related to the surface char- 
TABLE 1. AMSU-A characteristics.

\begin{tabular}{ccccc}
\hline \hline Channel No. & $\begin{array}{c}\text { Frequency } \\
(\mathrm{GHz})\end{array}$ & $\begin{array}{c}\text { Polarization } \\
\text { at nadir }\end{array}$ & $\begin{array}{c}\text { Atmospheric transmission } \\
\text { (tropical) }\end{array}$ & $\begin{array}{c}\text { Atmospheric transmission } \\
\text { (winter subarctic) }\end{array}$ \\
\hline 1 & 23.8 & $\mathrm{~V}$ & 0.78 & 0.99 \\
2 & 31.4 & $\mathrm{~V}$ & 0.89 & 0.96 \\
3 & 50.3 & $\mathrm{~V}$ & 0.63 & 0.68 \\
4 & 52.8 & $\mathrm{~V}$ & 0.29 & 0.32 \\
5 & $53.596 \pm 0.115$ & $\mathrm{H}$ & 0.11 & 0.13 \\
6 & 54.40 & $\mathrm{H}$ & 0.02 & 0.02 \\
7 & 54.94 & $\mathrm{H}$ & 0.00 & 0.00 \\
8 & 55.50 & $\mathrm{H}$ & 0.00 & 0.00 \\
9 & $57.290=\nu$ & $\mathrm{H}$ & 0.00 & 0.00 \\
10 & $\nu \pm 0.217$ & $\mathrm{H}$ & 0.00 & 0.00 \\
11 & $\nu \pm 0.322 \pm 0.048$ & $\mathrm{H}$ & 0.00 & 0.00 \\
12 & $\nu \pm 0.322 \pm 0.022$ & $\mathrm{H}$ & 0.00 & 0.00 \\
13 & $\nu \pm 0.322 \pm 0.010$ & $\mathrm{H}$ & 0.00 & 0.00 \\
14 & $\nu \pm 0.322 \pm 0.0045$ & $\mathrm{~V}$ & 0.00 & 0.00 \\
15 & 89.0 & 0.61 & 0.91 \\
\hline
\end{tabular}

acteristics. Holes in the map are due to the lack of clear-sky observations for this month for the observations with the given incidence angle range (the tropical band is particularly affected). As predicted by the models, the emissivities are higher in vegetated areas (e.g., the Siberian forest) than in arid region (e.g., the North African deserts and the Arabic Peninsula). Low emissivities are associated with areas of standing water or of highly saturated soils (north of Canada, and areas around the $\mathrm{Ob}$ River in Siberia or the Parana River in South America). Frequency and angular variations seem limited (the maps are similar), except for the 50.3$\mathrm{GHz}$ map for the high-incidence angle. Further analysis of these frequency and angular variations is performed in the next section.

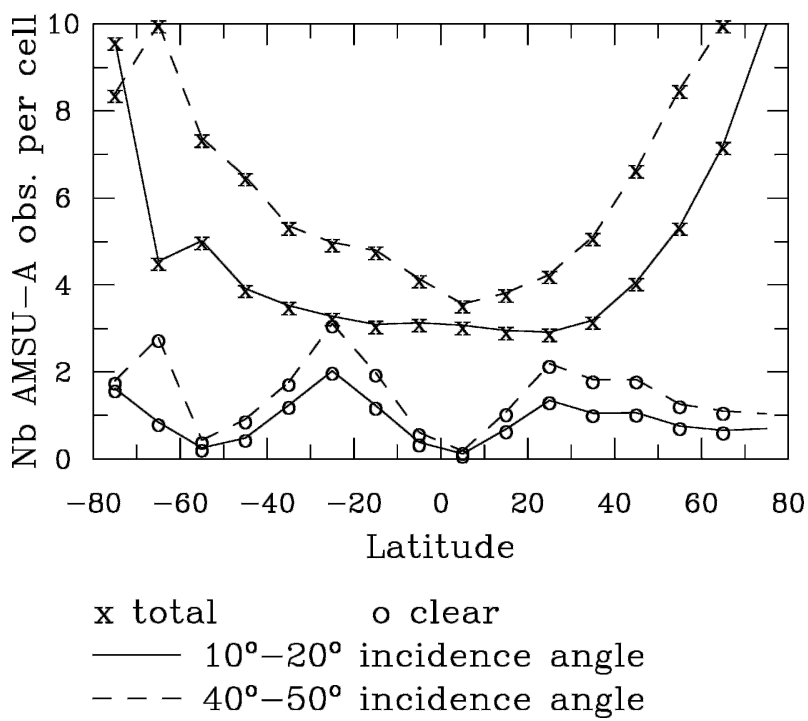

FIG. 2. The average number of AMSU-A observations per grid cell for two incidence angle ranges $\left(10^{\circ}-20^{\circ}\right.$ and $\left.40^{\circ}-50^{\circ}\right)$, for clear and cloudy scenes together $(\mathrm{x})$ and for cloud-free observations only (o). Results are presented for Jul 2002.

\section{Analysis and evaluation of the emissivity calculations-Comparison with other emissivity estimates}

Because of the lack of direct information about the surface emissivity at these relatively large spatial scales, one can only check that the expected behaviors are found. Evaluation strategy will be twofold: 1) to carefully check the consistency of the retrieved AMSU-A emissivities among themselves by verifying their frequency and angular dependencies and 2) to compare them with other microwave emissivities estimated at global scale.

For comparison purposes, two other emissivity calculations have been performed at ECMWF for the same 2 months. First, the SSM/I emissivities have been calculated, using the same radiative transfer code and the same ECMWF input data. Because SSM/I is a conical scanner, the incidence angle is fixed and each scene is observed more often under the same measurement conditions (see Fig. 2 in Prigent et al. 1997), reducing the noise in monthly mean estimates of the surface emissivity for a given location. Assuming that the frequency and angular dependencies of the emissivities are well known, estimates of the AMSU-A emissivities could be derived from the SSM/I emissivities. The frequency and angular intraextrapolation schemes previously developed (Prigent et al. 2000) will be tested here. Second, the Weng et al. (2001) emissivity model has also been implemented at ECMWF and will be compared.

The vegetation classification from Matthews (1983) is selected to sort the data by vegetation types. It is compiled from a large number of published sources and is independent of the datasets used here. The vegetation data distinguishes 30 classes, which are further grouped.

The angular dependence of the directly calculated AMSU-A emissivities (section 2) is first analyzed. For four surface types, Fig. 4 shows the mean emissivities 

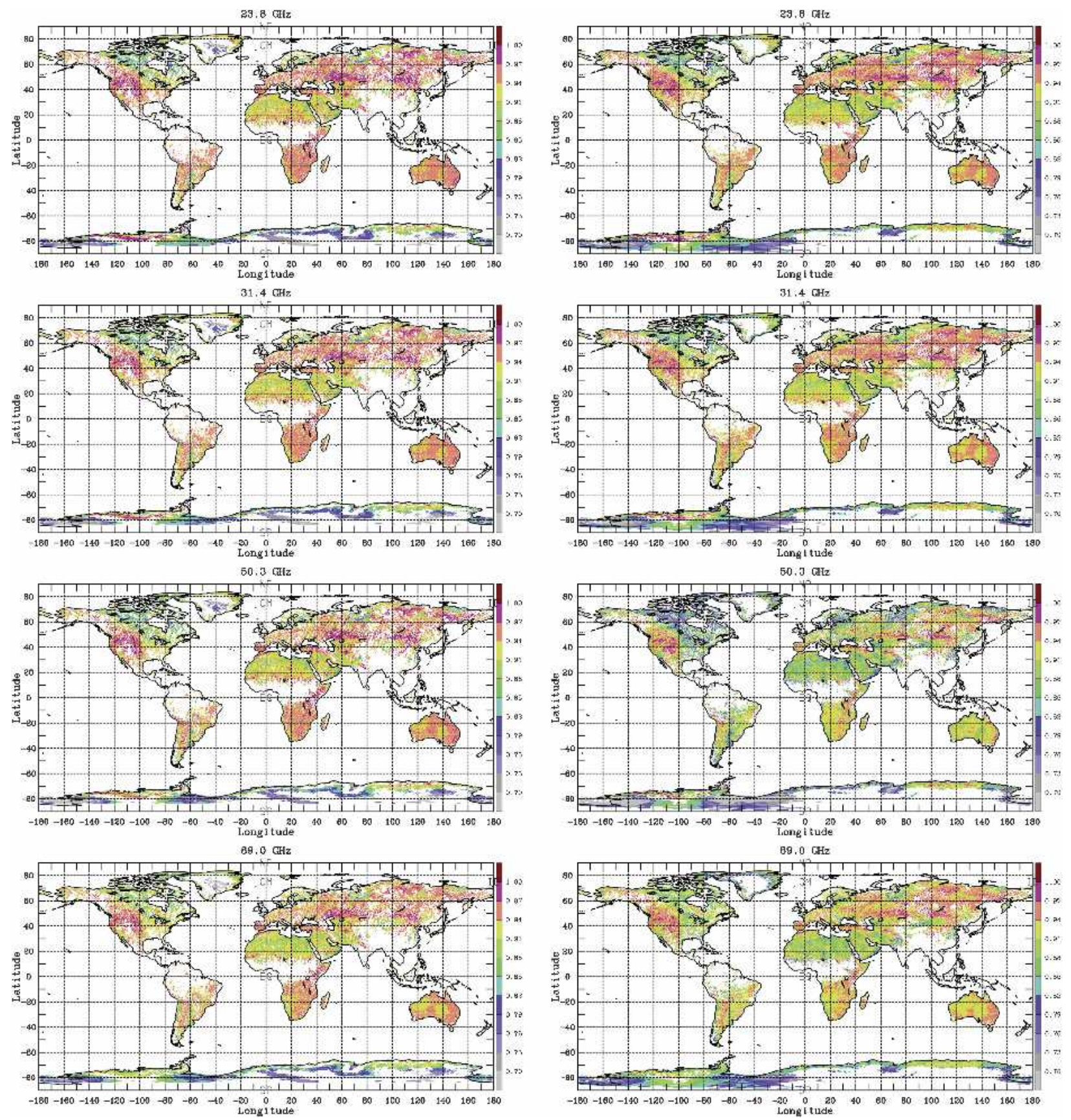

FIG. 3. Monthly mean emissivity maps for Jul 2002 for four AMSU-A channel frequencies for two incidence angle ranges: (left) $10^{\circ}-20^{\circ}$ and (right) $40^{\circ}-50^{\circ}$.

and their standard deviation for the four AMSU-A window channel frequencies for six ranges of incidence angles for January 2003. For each angular range, the monthly mean emissivity values are presented, along with their standard deviations over the month. The emissivities do not vary significantly with incidence angle up to $\sim 40^{\circ}$, and then they decrease with increasing angle. Given the polarization features of the AMSU-A observations, this is fully compatible with model simulations. In the same figure, comparison is provided with the angular and frequency fits derived from the corresponding SSM/I emissivity calculation performed at ECMWF for the same month (the middle solid line indicates the mean with the two others giving the standard deviation). The emissivity angular fit (Prigent et al. 2000) was derived from simulations for various surface types with the radiative transfer (RADTRAN) model (Isaacs et al. 1989). The figure 

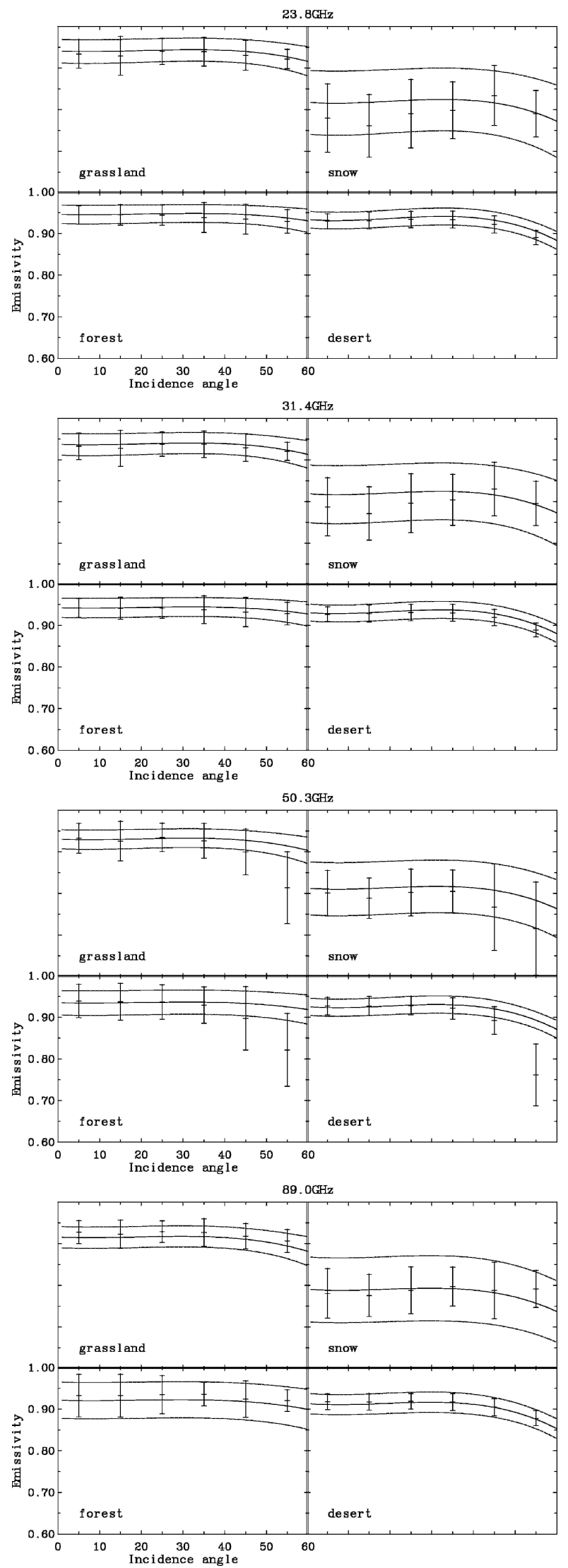

clearly shows that the expected angular behavior is obtained, except for the $50.3-\mathrm{GHz}$ channel for large angles. One reason can at least partly explain the discrepancy at $50.3 \mathrm{GHz}$. Except for some tropical atmospheres, the opacity is stronger at $50.3 \mathrm{GHz}$ than at the other frequencies presented in Fig. 4 (for a standard atmosphere, at nadir, the opacity is 0.62 at $50.3 \mathrm{GHz}$ and 0.30 at $89 \mathrm{GHz}$ ). First, this means that errors associated with the specular approximation are larger at this frequency for the large angles (see Fig. 1). Second, for a given error in the atmospheric profile or/and in the atmospheric absorption model, the errors will be larger, especially for large angles (see the sensitivity tests in Prigent et al. 2000). Over snow, the emissivities are more variable (larger standard deviations). They are sensitive to snow physical properties; interaction of the microwave radiation with snow involves volume scattering, especially for dry snow at high frequencies (Matzler 1994). Similar behaviors are observed in July (not shown).

Figure 5 presents the frequency dependence of the AMSU-A directly calculated emissivities for the four window channels and for four surface types, as calculated for July 2002, along with their standard deviations over the month. Only data for NOAA-16 with viewing angles smaller than $40^{\circ}$ are shown. The distinction is made between local nighttime and daytime observations, because the NOAA-16 spacecraft crosses the equator at about 0130/1330 LST. For comparison, the platforms that carry the SSM/I instruments cross the equator between 0530 and 0930 and between 1730 and 2130 LST.

As expected, for snow-free surface types, the frequency dependence of the emissivities as derived from the AMSU estimates is limited, with the emissivities slightly decreasing with increasing frequencies. This is very important-it means that interpolation/extrapolation of the emissivity calculation from the AMSU window channels to the AMSU sounding channels is possible. The frequency dependence estimated from the SSM/I-derived emissivities (as extrapolated in angle and frequencies to AMSU conditions) is more pronounced. However, one will note that the agreement between the SSM/I-extrapolated values and the AMSU at $50.3 \mathrm{GHz}$ is especially good for this broad angular range, whatever the surface. This is very encouraging for the extrapolation to the emissivities for the 50-60$\mathrm{GHz} \mathrm{O}_{2}$ channels. Comparison between the night- and

$\leftarrow$

FIG. 4. Angular dependence of the AMSU-A monthly mean emissivities, as directly calculated for Jan 2003, separated per surface types, for the four window channels. The standard deviations are indicated for each mean value (error bars). A comparison is provided with the angular and frequency fits derived from the corresponding SSM/I emissivity calculation performed at ECMWF for the same month (the middle solid line indicates the mean, with the two others giving the std dev). The emissivity and the incidence angle labels are the same for all boxes. 
(a) grassland 1
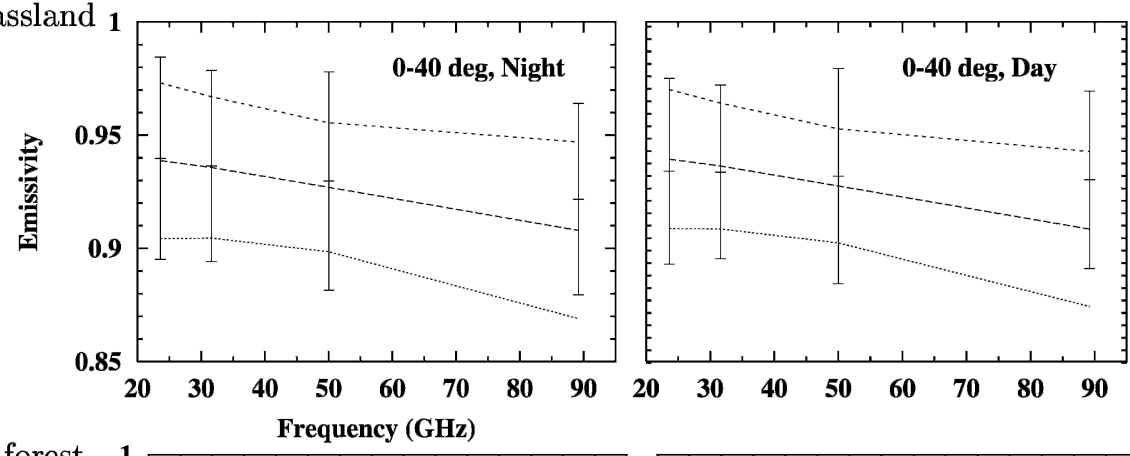

(b) forest
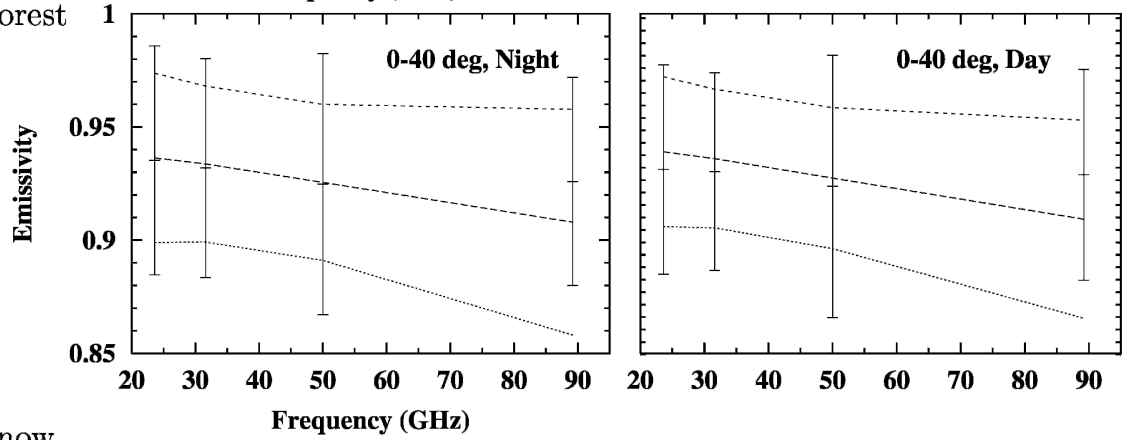

(c) snow

Frequency (GHz)
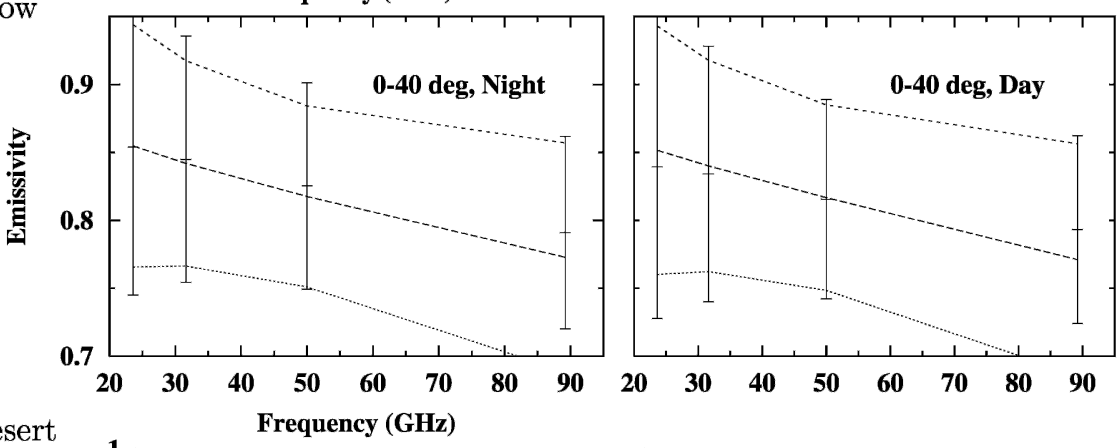

(d) desert
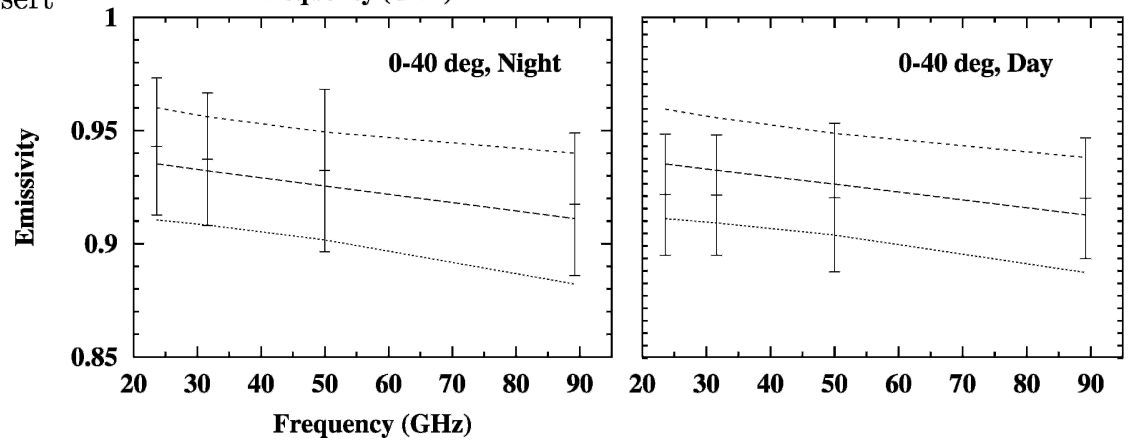

FIG. 5. Frequency dependence of the NOAA-16 AMSU-A monthly mean emissivities, as directly calculated for Jul 2002 , for incidence angles smaller than $40^{\circ}$, separated per surface types. The standard deviations are indicated for each mean value (error bars). A comparison is provided with the angular and frequency fits derived from the corresponding SSM/I emissivity calculation performed at ECMWF for the same month (the middle solid line indicates the mean, with the two others giving the std dev). The distinction is made between local night- and daytime observations. (a) Grassland, (b) forest, (c) snow, and (d) desert areas.

daytime NOAA-16 emissivity estimates shows a similar frequency behavior, except over desert surfaces. The lower the frequency, the larger the difference between the night- and daytime estimates, with a mean differ- ence of $\sim 0.02$ at $23.8 \mathrm{GHz}$. This can be explained by the different penetration depth of the radiation in a dry sand desert. In our calculation [Eq. (1)], the radiation is expected to emerge from a very thin surface layer that 
radiates according to the physical temperature of a thin layer at the surface (the skin temperature). However, in dry sand deserts, the microwave radiation might come from below the surface-the lower the frequency, the larger the penetration depth. In sand deserts, having a limited thermal inertia, strong thermal vertical gradients are observed below the surface, along with a large diurnal cycle. As a consequence, the "effective" emissivities that are calculated with the skin temperature vary during the diurnal cycle. This phenomenon had been analyzed in detail at SSM/I frequencies (Prigent et al. 1999). In addition, residual differences between the night- and daytime estimates for frequencies and regions where penetration of the radiation is not expected (e.g., at $89 \mathrm{GHz}$ over grassland) can also be related to an inadequate modeling of the surface skin temperature diurnal cycle in the ECMWF forecast model (Trigo and Viterbo 2003).

Differences between the AMSU-A directly calculated emissivities and the emissivities derived from SSM/I calculations are quantified in Table 2. Results include July and January values for the four window channels and for two incidence angle ranges, separated per vegetation types. Mean differences are indicated along with the rms difference (in parentheses). Biases are $\sim 1 \%$ or below for all surface types, even for snow, with rms values $\sim 0.02$ (slightly larger for snow). The behavior of the $50.3-\mathrm{GHz}$ channel for large angles is still the exception.

Monthly mean emissivity atlases have been calculated at the National Aeronautics and Space Administration (NASA) Goddard Institute for Space Studies (GISS) for the SSM/I frequencies and observations conditions for several years. From a practical point of view, it would be convenient to be able to interextrapolate these emissivities to the AMSU-A conditions, using the frequency and angular fit developed (Prigent et al. 2000). Direct calculations from AMSU-A have been compared with the interextrapolated SSM/I emissivities previously calculated at NASA GISS for July 1992 and January 1993. The results are presented on Table 3. Although the SSM/I emissivities were calculated using different inputs [NCEP-National Center for Atmospheric Research (NCAR) reanalysis (Kalnay et al. 1996) and ISCCP data] and a different radiative transfer code, and although interannual variability of the surface characteristics influences the emissivities, the overall results are very similar to the results given in Table 2. Not surprisingly, the rms errors are slightly larger, especially for the snow that can show large interannual variability in extent and in physical properties. This is clearly seen at $89 \mathrm{GHz}$, which is particularly sensitive to snow properties.

Last, the AMSU-A directly calculated emissivities are compared with the simulations of the Weng et al. (2001) emissivity model using the forecast modelrelevant surface variables (soil temperature and humidity, vegetation fraction, and snow depth) in input. The results are presented on Table 4 . The agreement with the SSM/I-based estimation is less good than for the previous results in Tables 2 and 3, likely because of both the simplicity of the ECMWF land surface model and that of the emissivity model. This is particularly true for desert and snow surfaces where most biases are larger than $1 \%$. Weng et al. (2001) already noticed the limitations of the snow emissivity model.

\section{Discussion and conclusions}

Over land surfaces, the utilization of passive microwave observations is usually limited to mid- and uppertropospheric sounding channels because of the lack of good surface emission modeling. At ECMWF, AMSU-A data that are affected by the surface (channels 5 and 6 at $53.6 \pm 0.115$ and $54.4 \mathrm{GHz}$ ) are, therefore, assimilated conservatively, assuming constant surface emissivities associated with several coarsely defined surface types. The surface-type classification employs AMSU-A window channels at 23.8, 31.4, 50.3, and $89.0 \mathrm{GHz}$ and does not take into account the variation of emissivity with scan angle.

In this paper, a new approach for extending the data usage over land was presented that provides a more dynamical emissivity estimate as a function of the local states of atmosphere and surface, respectively. Surface emissivity is derived using the ECMWF background information on surface skin temperature as well as atmospheric temperature and moisture profiles with observations at those frequencies that are not used in the assimilation. Two options are identified in which 1) the inversion is carried out at only one frequency (50.3

TABLE 2. Difference between AMSU-A emissivities directly calculated and emissivities estimated from inter-/extrapolations of the SSM/I emissivities calculated at ECMWF for the same months and years. Biases are indicated along with rms values (in parentheses).

\begin{tabular}{|c|c|c|c|c|c|c|}
\hline Frequency (GHz) & Angle & Forest & Grassland & Desert & Snow & Tot \\
\hline \multirow[t]{2}{*}{23.80} & $15^{\circ}$ & $-0.003(0.014)$ & $0.000(0.012)$ & $-0.001(0.012)$ & $-0.011(0.029)$ & $-0.004(0.019)$ \\
\hline & $45^{\circ}$ & $-0.011(0.021)$ & $0.008(0.017)$ & $-0.011(0.018)$ & $-0.005(0.018)$ & $-0.009(0.019)$ \\
\hline \multirow[t]{2}{*}{31.42} & $15^{\circ}$ & $-0.002(0.014)$ & $0.000(0.012)$ & $-0.002(0.012)$ & $-0.007(0.027)$ & $-0.002(0.018)$ \\
\hline & $45^{\circ}$ & $-0.009(0.021)$ & $-0.006(0.017)$ & $-0.009(0.017)$ & $-0.004(0.020)$ & $-0.007(0.018)$ \\
\hline \multirow[t]{2}{*}{50.33} & $15^{\circ}$ & $-0.002(0.022)$ & $0.006(0.020)$ & $-0.003(0.017)$ & $0.000(0.028)$ & $0.003(0.022)$ \\
\hline & $45^{\circ}$ & $-0.036(0.063)$ & $-0.027(0.051)$ & $-0.031(0.044)$ & $-0.060(0.098)$ & $-0.040(0.066)$ \\
\hline \multirow[t]{2}{*}{89.00} & $15^{\circ}$ & $0.013(0.023)$ & $0.014(0.024)$ & 0.006 (0.017) & $0.004(0.032)$ & $0.009(0.025)$ \\
\hline & $45^{\circ}$ & $0.004(0.024)$ & $0.007(0.023)$ & $-0.001(0.018)$ & $0.004(0.032)$ & $0.004(0.025)$ \\
\hline
\end{tabular}


TABLE 3. Difference between AMSU-A emissivities directly calculated and emissivities estimated from inter-/extrapolations of the SSM/I emissivities previously calculated at NASA GISS for the same months but different years. Biases are indicated, along with rms values (in parentheses).

\begin{tabular}{|c|c|c|c|c|c|c|}
\hline Frequency $(\mathrm{GHz})$ & Angle & Forest & Grassland & Desert & Snow & Tot \\
\hline \multirow[t]{2}{*}{23.80} & $15^{\circ}$ & $-0.002(0.022)$ & $0.001(0.023)$ & $-0.001(0.016)$ & $-0.012(0.041)$ & $-0.003(0.028)$ \\
\hline & $45^{\circ}$ & $-0.011(0.027)$ & $-0.005(0.024)$ & $-0.008(0.019)$ & $-0.010(0.034)$ & $-0.008(0.026)$ \\
\hline \multirow[t]{2}{*}{31.42} & $15^{\circ}$ & $0.000(0.022)$ & $0.004(0.023)$ & $0.002(0.015)$ & $-0.009(0.040)$ & $-0.001(0.028)$ \\
\hline & $45^{\circ}$ & $-0.009(0.026)$ & $-0.001(0.024)$ & $-0.006(0.018)$ & $-0.009(0.038)$ & $-0.006(0.028)$ \\
\hline \multirow[t]{2}{*}{50.33} & $15^{\circ}$ & $0.000(0.028)$ & $0.007(0.033)$ & $0.003(0.021)$ & $-0.005(0.046)$ & $0.001(0.035)$ \\
\hline & $45^{\circ}$ & $-0.042(0.073)$ & $-0.025(0.055)$ & $-0.026(0.042)$ & $-0.070(0.117)$ & $-0.041(0.079)$ \\
\hline \multirow[t]{2}{*}{89.00} & $15^{\circ}$ & $-0.002(0.025)$ & $0.006(0.038)$ & $-0.004(0.022)$ & $0.013(0.065)$ & $0.003(0.045)$ \\
\hline & $45^{\circ}$ & $-0.012(0.032)$ & $0.000(0.042)$ & $-0.009(0.025)$ & $-0.014(0.070)$ & $-0.008(0.049)$ \\
\hline
\end{tabular}

$\mathrm{GHz}$ ), assuming that the emissivity is identical to that at 52.8, $53.6 \pm 0.115$, and $54.4 \mathrm{GHz}$, and 2) emissivities are derived separately at $23.8,31.4$, and $89.0 \mathrm{GHz}$ and are linearly interpolated to the frequency of choice. The advantage of the second option is the much smaller atmospheric opacity in the window channels. This is important because errors in the background information may alias the surface emissivity estimate and therefore negatively affect the analysis. However, the interpolation over frequencies and the difference in polarization variation across the scan between window and sounding channels introduce uncertainties as well.

Surface emissivities have been calculated at 23.8, 31.4, 50.3, and 89.0 GHz for 2 months and compared with other satellite products because of the lack of independent validation data. It was found that both the angular variation and frequency dependence of emissivity were small for zenith angles below $40^{\circ}$ and over all surface types except snow-covered regions. Also, surfaces that show distinct volume scattering (dry soil and sand) exhibited a larger dispersion in the comparison as a function of daytime.

Differences between the two products were found to be small and mainly associated with the abovementioned scattering surface types, that is, sand and snow, and scan position (at $50.3 \mathrm{GHz}$ only). Another possibility of evaluation was exploited that is the forward calculation of emissivity using physical surface and vegetation parameters available from the ECMWF model parameterizations, such as soil type, soil moisture, vegetation coverage, and water content. In this case, the derived and modeled emissivities showed larger discrepancies that can be interpreted as short- comings in emissivity modeling and the limited accuracy of the input parameters.

The final means of evaluation is the test of the scheme in the ECMWF data assimilation context. For this purpose, two experiments were done, a control experiment with the operational configuration and an experiment that employed the derivation of emissivity at the window frequencies with interpolation to the sounding frequencies. Two model cycles were performed on 18 July 2003, at 0000 and 1200 UTC. In the control experiment, AMSU-A channel $4(52.8 \mathrm{GHz})$ was not used in the assimilation, but was used for data screening. In the emissivity experiment, this channel was activated as well.

Over land, a total number of 410309 AMSU-A observations were counted of which 0,18 602, and 23634 were used in the minimization of the control experiment for channels 4,5 , and 6 , respectively. In the emissivity experiment these numbers increased to 26383,22 174, and 27 712, which is an increase by $17 \%-19 \%$ in channels 5 and 6 , because more data passed the quality control (which is based on observations minus calculations). This increase of observation numbers is very important. The fact that more observations are assimilated than in the control experiment clearly indicates an improvement of the forward modeling because the fit to the observations has been improved. The introduction of channel 4 modifies the analyzed temperature by about $0.4 \mathrm{~K}$ (rms difference) in the lower troposphere over land in the first analysis cycle. The differences reach up to several degrees locally and grow a bit larger in the subsequent cycles. However, the impact on the forecast was small and, overall, neutral. It has to be

TABle 4. Difference between AMSU-A emissivities directly calculated and emissivities simulated with Weng et al. (2001) model. Biases are indicated, along with rms values (in parentheses).

\begin{tabular}{ccccrrr}
\hline \hline Frequency $(\mathrm{GHz})$ & Angle & Forest & Grassland & \multicolumn{1}{c}{ Desert } & \multicolumn{1}{c}{ Snow } & Tot \\
\hline 23.80 & $15^{\circ}$ & $0.015(0.038)$ & $0.008(0.033)$ & $-0.004(0.025)$ & $-0.099(0.140)$ & $-0.023(0.077)$ \\
& $45^{\circ}$ & $0.008(0.044)$ & $0.000(0.037)$ & $-0.014(0.033)$ & $-0.053(0.091)$ & $-0.015(0.055)$ \\
31.42 & $15^{\circ}$ & $0.006(0.034)$ & $0.000(0.031)$ & $-0.009(0.026)$ & $-0.070(0.103)$ & $-0.021(0.059)$ \\
& $45^{\circ}$ & $0.000(0.041)$ & $-0.006(0.035)$ & $-0.017(0.034)$ & $-0.034(0.069)$ & $-0.015(0.046)$ \\
50.33 & $15^{\circ}$ & $-0.009(0.043)$ & $-0.010(0.036)$ & $-0.011(0.031)$ & $0.131(0.162)$ & $0.027(0.089)$ \\
& $45^{\circ}$ & $-0.046(0.087)$ & $-0.044(0.073)$ & $-0.045(.065)$ & $0.083(0.175)$ & $-0.014(0.107)$ \\
89.00 & $15^{\circ}$ & $-0.018(0.039)$ & $-0.020(0.037)$ & $0.021(0.058)$ & $0.507(0.546)$ & $0.132(0.284)$ \\
& $45^{\circ}$ & $-0.025(0.032)$ & $-0.029(0.042)$ & $-0.016(0.025)$ & $0.463(0.521)$ & $0.101(0.258)$ \\
\hline
\end{tabular}


noted that data screening and observation error quantification treatment remained unchanged. In the future, these would have to be revised for an optimum utilization of the information contained in the additional observations that are sensitive to the lower troposphere over land.

Acknowledgments. We thank Jean-Noel Thépaut (ECMWF) for his support and for his careful review of an earlier version of the paper. We are very grateful to Stephen English and two other anonymous reviewers for detailed and constructive comments on the manuscript. Part of this work was done in the context of the Satellite Application Facility on Numerical Weather Prediction, which is cosponsored by EUMETSAT.

\section{REFERENCES}

Aires, F., C. Prigent, W. B. Rossow, and M. Rothstein, 2001: A new neural network approach including first-guess for retrieval of atmospheric water vapor, cloud liquid water path, surface temperature and emissivities over land from satellite microwave observations. J. Geophys. Res., 106, 14 887-14 907.

Calvet, J.-C., A. Chanzy, and J.-P. Wigneron, 1996: Surface temperature and soil moisture retrieval in the Sahel from airborne multifrequency microwave radiometry. IEEE Trans. Geosci. Remote Sens., 34, 588-600.

Chevallier, F., and G. Kelly, 2002: Model clouds as seen from space: Comparison with geostationary imagery in the $11-\mu \mathrm{m}$ window channel. Mon. Wea. Rev., 130, 712-722.

Deblonde, G., 2000: Evaluation of FASTEM and FASTEM2. Met Office NWP SAF Rep. NWPSAF-MO-VS-001, 33 pp.

Diak, G. R., D. Kim, M. S. Whipple, and X. Wu, 1992: Preparing for the AMSU. Bull. Amer. Meteor. Soc., 73, 1971-1984.

English, S. J., 1999: Estimation of temperature and humidity profile information from microwave radiances over different surface types. J. Appl. Meteor., 38, 1526-1541.

_ , and T. Hewison, 1998: A fast generic millimeter-wave emissivity model. Microwave Remote Sensing of the Atmosphere and Environment, T. Hayasaka et al., Eds., International Society for Optical Engineering (SPIE Vol. 3503), 288-300.

, R. J. Renshaw, P. C. Dibben, A. J. Smith, P. J. Rayer, C. Poulsen, F. W. Saunders, and J. R. Eyre, 2000: A comparison of the impact of TOVS and ATOVS satellite sounding data on the accuracy of numerical weather forecasts. Quart. J. Roy. Meteor. Soc., 126, 2911-2931.

Eyre, J. R., 1991: A fast radiative transfer model for satellite sounding systems. ECMWF Tech. Memo. 176, 28 pp.

Felde, G. W., and J. D. Pickle, 1995: Retrieval of 91 and $150 \mathrm{GHz}$ Earth surface emissivities. J. Geophys. Res., 100, 20855 20866.

Ferrazoli, P., J.-P. Wigneron, L. Guerriero, and A. Chanzi, 2000: Multifrequency emission of wheat: Modeling and applications. IEEE Trans. Geosci. Remote Sens., 38, 2598-2607.

Fily, M., A. Royer, K. Goita, and C. Prigent, 2003: A simple retrieval method for land surface temperature and fraction of water surface determination from satellite microwave brightness temperatures in sub-arctic areas. Remote Sens. Environ., $\mathbf{8 5}, 328-338$.

Fung, A. K., 1994: Microwave Scattering and Emission Models and Their Applications. Artech House, 573 pp.

Grody, N. C., 1988: Surface identification using satellite microwave radiometers. IEEE Trans. Geosci. Remote Sens., 26, $850-859$.

Hewison, T. J., 2001: Airborne measurements of forest and agricultural land surface emissivity at millimeter wavelengths. IEEE Trans. Geosci. Remote Sens., 39, 393-400.

- and S. J. English, 1999: Airborne retrievals of snow and ice surface emissivity at millimeter wavelength. IEEE Trans. Geosci. Remote Sens., 37, 1871-1879.

Isaacs, R. G., Y.-Q. Jin, R. D. Worsham, G. Deblonde, and V. J. Falcone, 1989: The RADTRAN microwave surface emission models. IEEE Trans. Geosci. Remote Sens., 27, 433-440.

Jones, A. S., and T. H. Vonder Haar, 1997: Retrieval of surface emittance over land using coincident microwave and infrared satellite measurements. J. Geophys. Res., 102, 13 609-13 626.

Kalnay, E., and Coauthors, 1996: The NCEP/NCAR 40-Year Reanalysis Project. Bull. Amer. Meteor. Soc., 77, 437-471.

Karam, M. A., A. K. Fung, R. H. Lang, and N. S. Chuahan, 1992: A microwave scattering model for layered vegetation. IEEE Trans. Geosci. Remote Sens., 30, 767-784.

Kelly, G., and P. Bauer, 2000: The use of AMSU-A surface channels to obtain surface emissivity over land, snow and ice for Numerical Weather Prediction. Proc. 11th Int. TOVS Study Conf., Budapest, Hungary, 167-179.

Matthews, E., 1983: Global vegetation and land use: New highresolution data bases for climate studies. J. Climate Appl. Meteor., 22, 474-486.

Matzler, C., 1990: Seasonal evolution of microwave radiation from an oat field. Remote Sens. Environ., 31, 161-173.

_ 1994: Passive microwave signatures of landscapes in winter. Meteor. Atmos. Phys., 54, 241-260.

Prigent, C., W. B. Rossow, and E. Matthews, 1997: Microwave land surface emissivities estimated from SSM/I observations. J. Geophys. Res., 102, 21 867-21 890.

,$- \ldots$, and _ 1998: Global maps of microwave land surface emissivities: Potential for land surface characterization. Radio Sci., 33, 745-751.

$[, \ldots$, and —_, 1999: Microwave radiometric signatures over different surface types in deserts. J. Geophys. Res., 104, $12147-12158$

— J. P. Wigneron, W. B. Rossow, and J. R. Pardo-Carrion, 2000: Frequency and angular variations of land surface microwave emissivities: Can we estimate SSM/T and AMSU emissivities from SSM/I emissivities? IEEE Trans. Geosci. Remote Sens., 38, 2373-2386.

_ , F. Aires, W. B. Rossow, and E. Matthews, 2001a: Joint characterization of vegetation by satellite observations from visible to microwave wavelength: A sensitivity analysis. $J$. Geophys. Res., 106, 20 665-20 685.

_ E. Matthews, F. Aires, and W. B. Rossow, 2001b: Remote sensing of global wetland dynamics with multiple satellite data sets. Geophys. Res. Lett., 28, 4631-4634.

_, F. Aires, and W. B. Rossow, 2003: Land surface skin temperatures from a combined analysis of microwave and infrared satellite observations for an all-weather evaluation of the differences between air and skin temperatures. J. Geophys. Res., 108, 4310, doi:10.1029/2002JD002301.

Rossow, W. B., and R. A. Schiffer, 1999: Advances in understanding clouds from ISCCP. Bull. Amer. Meteor. Soc., 80, 2261-2287.

Saunders, R. W., 1993: Note on the Advanced Microwave Sounding Unit. Bull. Amer. Meteor. Soc., 74, 2211-2212.

_ M. Matricardi, and P. Brunel, 1999: An improved fast radiative transfer model for assimilation of satellite radiance observations. Quart. J. Roy. Meteor. Soc., 125, 1407-1425.

Shi, J., K. S. Chen, Q. Li, T. J. Jackson, and P. E. O'Neil, 2002: A parameterized surface reflectivity model and estimation of bare-surface soil moisture with L-band radiometer. IEEE Trans. Geosci. Remote Sens., 40, 2674-2686.

Trigo, I. F., and P. Viterbo, 2003: A comparison between observations and the ECMWF model. J. Appl. Meteor., 42, 1463-1479.

Weng, F., B. Yan, and N. C. Grody, 2001: A microwave land emissivity model. J. Geophys. Res., 106, 20 115-20 123.

Wigneron, J.-P., D. Guyon, J.-C. Calvet, G. Courrier, and N. Bruguier, 1997: Monitoring coniferous forest characteristics using a multifrequency $(5-90 \mathrm{GHz})$ microwave radiometer. Remote Sens. Environ., 60, 299-310. 